\title{
Kontext und KI: Zum Potenzial der Beschäftigten für Künstliche Intelligenz und Machine-Learning
}

\author{
Sabine Pfeiffer $(\mathbb{D}$
}

Eingegangen: 31. Dezember 2019 / Angenommen: 10. März 2020 / Online publiziert: 20. März 2020 (C) Der/die Autor(en) 2020

Zusammenfassung Die Bedeutung von Künstlicher Intelligenz und Machine Learning (KI/ML) im Unternehmen nimmt zu. Noch weitgehend unklar ist, ob das Potenzial der Beschäftigten ausreicht und passfähig ist, um die Potenziale dieser Technologie im Unternehmen schnell nutzbar zu machen. Neben den dafür nötigen KI/MLspezifischen Programmierkenntnissen in der IT-Abteilung erfordert ein robuster und produktiver Einsatz von KI/ML im Unternehmen auch von den Beschäftigten in den anwendenden Fachabteilungen neue Kompetenzen. Sie müssen die Potenziale und Grenzen von KI/ML-Technologien verstehen (KI/ML-Kompetenz) und in der Lage sein, KI/ML-Systeme und ihre Ergebnisse in den fachlichen Kontext und an situative Anforderungen rückzubinden (Kontext-Kompetenz). Der Beitrag identifiziert diese Kompetenzen und zeigt empirisch, in welchem Ausmaß sie vorhanden sind oder entwickelt werden müssen. Dies geschieht auf der Basis der BIBB/BAuAErwerbstätigenbefragung 2018 für zwei Felder, die aktuell besonders relevant für den Einsatz von KI/ML sind: Predictive Maintenance und beratungsintensive Sachbearbeitung. Die Ergebnisse zeigen: Beschäftigte haben ein unterschätztes Potenzial für die erfolgreiche Umsetzung von KI/ML, es lohnt sich also, sie in allen Phasen der KI/ML-Umsetzung partizipativ zu involvieren.

Schlüsselwörter Vorausschauende Wartung · Personalwesen · Finanzdienstleistungen $\cdot$ Big Data $\cdot$ DataScience $\cdot$ Kompetenzen

\footnotetext{
S. Pfeiffer $(\bowtie)$

Nuremberg Campus of Technology (NCT), Friedrich-Alexander-Universität Erlangen-Nürnberg, Nürnberg, Deutschland

E-Mail: sabine.pfeiffer@fau.de
} 


\title{
Context and AI: On Employees' Potential for Artificial Intelligence and Machine Learning
}

\begin{abstract}
The importance of Artificial Intelligence and Machine Learning (AI/ML) in companies is increasing. However, it is largely unclear whether the employees' abilities are sufficient and suitable to make the potential of this technology rapidly usable in the company. In addition to the necessary AI/ML-specific programming knowledge in the IT department, a robust and productive use of AI/ML in the company also requires new skills from employees in the applying departments. They must understand the potential and limits of $\mathrm{AI} / \mathrm{ML}$ technologies (AI/ML competence) and they must be able to integrate $\mathrm{AI} / \mathrm{ML}$ systems and their results into the specific context and situational requirements (context competence). The article identifies these competencies and shows empirically to what extent they are available or need to be developed. This is done on the basis of the 2018 German BIBB/ BAuA Employment Survey for two areas of application that are currently considered particularly relevant for the use of AI/ML: Predictive maintenance and consulting intensive administration. The results show: Employees have an underestimated potential for a successful application of $\mathrm{AI} / \mathrm{ML}$, so it is worthwhile to involve them in all stages of $\mathrm{AI} / \mathrm{ML}$ implementation.
\end{abstract}

Keywords Predictive Maintenance $\cdot$ HR $\cdot$ Financial Services · Big Data · DataScience $\cdot$ Competencies

\section{KI und Kontext: Ein potenzialorientierter Blick}

Künstliche Intelligenz und Machine Learning (KI/ML) gelten als Technologien, die alle Bereiche der Arbeitswelt erfassen und letztlich entscheidend sind für fast alles, was seit einigen Jahren unter den Stichworten „Digitalisierung“ oder „4.0“ diskutiert wird. Üblicherweise wird das Verhältnis von Beschäftigten (bzw. den von ihnen ausgeübten Tätigkeiten) und neuen Formen der Digitalisierung dabei als ein passives diskutiert. Dieser Beitrag geht dagegen der Frage nach, ob und warum eine aktive Rolle der Beschäftigten für eine erfolgreiche Umsetzung von KI/ML notwendig ist. In den meisten quantitativen Prognosen (wie etwa Dengler und Matthes 2015; Helmrich et al. 2016; Frey und Osborne 2017) wird Digitalisierung als ein im Unternehmen sich bruchlos durchsetzender Fakt betrachtet - mit kausalen Auswirkungen auf menschliche Arbeit, die lediglich noch erforderlich machen, Ausmaß und Geschwindigkeit einzuschätzen. Wichtiger wäre aber zu fragen, ob und welcher Form menschliche Arbeit benötigt wird, um KI/ML zu einem Erfolgsmodell zu machen. Im Hinblick auf Arbeitsmarkteffekte wird KI/ML das Potenzial zugeschrieben, in Bereiche menschlichen Arbeitshandelns vorzudringen, die bisher als technisch nicht ersetzbar galten. Frey und Osborne etwa illustrieren diese Verschiebungsthese am Beispiel des autonomen Fahrens, das bei den früheren Studien von Levy und Murnane (2005) noch als nicht technisch ersetzbar eingeschätzt wurde. Dieser Beitrag fragt nach den Kompetenzen der Beschäftigten, die für eine sich dynamisch sich verändernde KI/ML-Anwendung im Unternehmen benötigt wird. 
Dieser Artikel zielt also auf den praktischen Einsatz von KI/ML in der Arbeitswelt, aber nicht mit der Perspektive auf Ersetzbarkeit, sondern mit der Frage danach, wie ein sinnvoller Einsatz von KI/ML in Unternehmen gestaltet werden kann und welche Potenziale die Beschäftigten dafür mitbringen. Führungskräfte sehen hier für die Umsetzung von KI/ML oft große Hürden und betrachten die eigenen Beschäftigten dabei oft mit einem Blick auf vermeintliche Defizite. Die nachfolgenden empirischen Untersuchungen drehen die Perspektive um und verfolgen eine fundierte Einschätzung zu den Potenzialen der Beschäftigten für eine erfolgreiche KI/MLUmsetzung.

Betrachtet werden dafür Anwendungsbereiche, die einerseits aktuell als besonders relevant für KI/ML-Anwendungen eingeschätzt werden und die sich andererseits auf Prozesse richten, die üblicherweise als wissens- und/oder beratungsintensiv betrachtet werden. Es stehen damit Bereiche im Fokus, in denen bislang die menschenund erfahrungsgebundene Facharbeits- oder Beratungsexpertise als unersetzbar galt. Exemplarisch kontrastiert werden Beschäftigtenkompetenzen und -potenziale in:

- einem stofflich und maschinell geprägtem Umfeld am Beispiel der vorausschauenden Wartung (Predictive Maintenance) in den Feldern Produktions- und Infrastrukturprozesse.

- der beratungsintensiven Sachbearbeitung in den unternehmensinternen Felder Personalwesen (HR) und. Unternehmensorganisation sowie in der kundennahe Beratung in Banken und Versicherungen ausgewählt.

In diesen Feldern drängen KI/ML-Anwendungen derzeit in die anwendende Unternehmenspraxis vor: So finden sich unterschiedlichste Ansätze intelligenter Instandhaltungssysteme für die Produktion einer Industrie 4.0 (Garcia et al. 2020), aber auch für Prozesstechnik wie der Energieversorgung (Doleski 2020) oder im Bereich von Infrastrukturanlagen (Timofeev und Denisov 2020). Im Personalbereich und Unternehmensorganisation reichen die Anwendung von der Rekrutierung (Tallgauer et al. 2020) über die Chatbot gestützte Beratung bis zur Voraussage von Burnout (Lemmer et al. 2019) oder von Personalfluktuation (Zhao et al. 2019). In Banken und Versicherungen werden beratungsintensive Bereiche über ein ,kognitives Dashboard“" unterstützt, unzufriedene Kund*innen durch Gesichts-/Sentiment-Analysen identifiziert oder ein RoboAdvisor für komplexe Anlagenberatungen eingesetzt (Zacherl et al. 2019). Für die Versicherungsbranche finden sich zudem Digitale Assistance-Dienstleistungen (Elert 2019) oder Frühwarnsysteme der Kundenbindung (Reich und Blodau 2019). Zunächst kategorisiert die empirische Betrachtung die genannten Beschäftigungsbereiche in quantitativen Erwerbsdaten (2. Kapitel).

Auf dieser Basis kann im nächsten Analyseschritt eine Einschätzung der Potenziale dieser Beschäftigtenbereiche für eine sinnvolle Umsetzung von KI/ML erfolgen. Üblicherweise werden die Anforderungen an Beschäftigte reduziert auf Data Science-Kompetenzen im technischen Sinne: So zeigt eine Auswertung von Stellenanzeigen (Debortoli et al. 2014), dass überwiegend technische Fähigkeiten nach Erfahrung mit NoSQL Databases und Software Engineering gesucht wird. Eine neuere Analyse (Zschech et al. 2018) nennt als Anforderungen an Data Scientists vor allem Erfahrung mit R, Python, SQL, Java sowie Hadoop SAS, MapReduce und SPSS. 
Hier wird ein anderer Ansatz verfolgt. Es wird davon ausgegangen, dass ein robuster und produktiver Einsatz von KI/ML im Unternehmen weit mehr und andere Kompetenzen der Beschäftigten braucht und dass diese Kompetenzen nicht nur in den entwickelnden Bereichen, sondern auch in den anwendenden Fachabteilungen benötigt werden und zwar bereits in den vier Prozessschritten der Umsetzung, laut Ng und Schoo (2018, S. 2-18) also von der Datenaufbereitung, über die Algorithmenauswahl und Parameteroptimierung bis zur Modellableitung inklusive Evaluierung und Validierung.

Alle vier Schritte erfordern einerseits IT- und elaborierte Statistik-Kenntnisse (KI-Kompetenz) und andererseits ein auf den Anwendungsbereich bezogenes, qualitatives Expertenwissen (Kontext-Kompetenz). Auch in der faktischen Nutzung bereits implementierter KI/ML-Systeme bleiben beide Kompetenzen weiterhin von Relevanz, denn ein in einem sich verändernden Unternehmensumfeld permanent weiterlernender Algorithmus erfordert immer wieder die Notwendigkeit einer ReKontextualisierung und der situativen Plausibilisierung seiner Ergebnisse. Ob und inwieweit Beschäftigte die dafür nötigen Kompetenzen bereits haben, beleuchtet mit aktuellen empirischen Daten das 3. Kapitel und zeigt damit auf, welche Potenziale für die Umsetzung von KI/ML bei den ausgewählten Beschäftigungsgruppen im Hinblick auf KI und auf Kontext vorhanden sind.

Basis für diese empirischen Schritte sind eigene Berechnungen mit den jüngst erst zugänglich gemachten Daten der BIBB/BAuA-Erwerbstätigenbefragung 2018. Dabei handelt es sich um eine seit 1979 wiederholt durchgeführte Repräsentativbefragung mit aktuell $N=20.012$ Erwerbstätigen in Deutschland zu den Themen Arbeit, Beruf und Qualifikation. Erfasst sind Kernerwerbstätige, die einer bezahlten Arbeit von mindestens zehn Wochenstunden nachgehen (Rohrbach-Schmidt und Hall 2020) ${ }^{1}$. Die Ergebnisse werden abschließend im 4. Kapitel vor dem Hintergrund der Grenzen von KI/ML-Anwendungen diskutiert, die im Unternehmen insbesondere auf Managementebene oft noch unterschätzt werden.

\section{Kontexte für KI/ML erfassen: Predictive Maintenance und beratungsintensive Sachbearbeitung}

Zwei Bereiche werden derzeit besonders häufig als Beispiele für aktuelle Anwendungen von KI/ML in Unternehmen genannt: Einerseits Predictive Maintenance und andererseits die Automatisierung von Entscheidungen im Bereich beratungsinrensiver Sachbearbeitung (wie sie etwa im Personalwesen sowie oder in der Beratung zu Bank- und Versicherungsprodukten vorkommt). Nachfolgend wird gezeigt, wie diese für den Einsatz von KI/ML betroffenen Beschäftigtengruppen statistisch kategorisiert werden können. Will man diese Beschäftigten aus dem Datensatz extrahieren, ist ein einfacher Zugriff allein über den Beruf nicht ausreichend, sondern muss ergänzt werden um das Anforderungsniveau und einschlägige Tätigkeiten.

\footnotetext{
${ }^{1}$ Für die Auswertungen wird das Scientific Use File (https://doi.org/10.7803/501.18.1.1.10) der Version 1.0 verwendet, das seit dem 13. Februar 2020 zugänglich ist.
} 


\subsection{Kontext Predictive Maintenance}

Ein erster Kategorisierungsschritt erfolgt über Berufe: Als Kontextbeschäftigte im Bereich Instandhaltung werden aus jeweils sieben Berufen nach der KldB2010 auf 3-Steller-Ebene zunächst zwei Berufsgruppen zusammengefasst: Die erste Berufsgruppe (MuE/Automotive) umfasst Metall- und Elektroberufe, die überwiegend im produzierenden Gewerbe vorkommen: Metallerzeugung (241), Metallbearbeitung (242), Maschinenbau- und Betriebstechnik (251), Fahrzeug-Luft-Raumfahrt-, Schiffbauberufe (252), Mechatronik/Automatisierung (261), Elektrotechnik (263) sowie Technische Produktionsplanung, -steuerung (273; insgesamt $N=1677)$. Die zweite Berufsgruppe (Prozesse/Infrastruktur) fasst Berufe zusammen, die sich stärker auf die Instandhaltung von Gebäuden, Infrastruktur sowie großtechnischen Anlagen beziehen: Energietechnik (262), Gebäudetechnik (341), Klempnerei, Sanitär, Heizung und Klimatechnik (342), Ver- und Entsorgung (343), Chemie (413), Überwachung, Wartung und Verkehrsinfrastruktur (512) Überwachung und Steuerung Verkehr (515; insgesamt $N=837)$. Im zweiten Kategorisierungsschritt wird eine weitere Eingrenzung über Tätigkeiten vorgenommen: Aus beiden Gruppen werden nur Fälle aufgenommen, die bei den Tätigkeiten angeben, häufig mit dem „Überwachen, Steuern von Maschinen, Anlagen etc.“ (F305) und häufig mit „Reparieren, Instandsetzen“ (F306), aber selbst nie mit dem „Herstellen, Produzieren von Waren und Gütern" (F303) beschäftigt zu sein. Für den dritten Kategorisierungsschritt ist das Anforderungsniveau entscheidend: Weil die Instandhaltung komplexer Anlagen üblicherweise ein Anforderungsprofil auf der Ebene beruflicher Erst- und/oder Weiterbildung erfordert und weil nur Beschäftigte erfasst werden sollten, die selbst konkret mit Instandhaltung befasst sind (statt diese bspw. zu planen), wurden zusätzlich nur die Fälle aufgenommen, die eine Anforderung (F400) auf Fachkraftoder Spezialist*innen-Niveau angaben, nicht solche auf dem Anforderungsniveau unterhalb eines beruflichen oder auf der Ebene eines akademischen Abschlusses.

Nach dieser dreifachen Kategorisierung umfassen die Kontextbeschäftigten in den beiden Instandhaltungsbereichen MuE/Automotive $N=138$ (nachfolgend $P M \_M A$ ) und Prozesse/Infrastruktur N=127 (nachfolgend $P M_{-} P I$ ). Beide Gruppen sind überwiegend männlich (97,8\% bei PM_MA und 94,5\% bei PM_PI), im Durchschnitt 43,4 (MA; $\mathrm{SD}=10,85)$ und 45,8 (PI; $\mathrm{SD}=12,20)$ Jahre alt und durchschnittlich seit 12,2 (MA; SD=9,82) bzw. 14 (PI; SD=9,89) Jahren in der aktuellen Tätigkeit

Tab. 1 Stichproben

\begin{tabular}{llllllllll}
\hline \multicolumn{2}{c}{ Geschlecht } & & \multicolumn{3}{c}{ Alter } & \multicolumn{5}{c}{ Erfahrungsjahre } \\
& $N$ & $\begin{array}{l}\text { Männlich } \\
(\%)\end{array}$ & $\begin{array}{l}\text { Weiblich } \\
(\%)\end{array}$ & $N$ & MW & SD & $N$ & MW & SD \\
\hline$P M \_M A$ & 138 & 97,80 & 2,20 & 135 & 43,36 & 10,854 & 126 & 12,02 & 9,815 \\
$P M \_P I$ & 127 & 94,50 & 5,50 & 123 & 45,76 & 12,164 & 120 & 14,08 & 11,152 \\
$B A \_P O$ & 392 & 36,00 & 64,00 & 386 & 46,57 & 10,238 & 370 & 9,89 & 8,265 \\
$B A \_B V$ & 310 & 48,40 & 51,60 & 300 & 47,12 & 9,755 & 236 & 13,14 & 9,455 \\
$D E$ & 20.012 & 50,34 & 49,66 & 19.523 & 46,83 & 11,044 & 17.439 & 10,56 & 9,465 \\
alle & & & & & & & & & \\
\hline
\end{tabular}


Tab. 2 Digitalisierung am Arbeitsplatz

\begin{tabular}{|c|c|c|c|c|c|c|c|c|}
\hline \multicolumn{5}{|c|}{ Arbeit mit Computer } & \multicolumn{4}{|c|}{ Arbeit mit Web/Email } \\
\hline & $N$ & $\begin{array}{l}\text { Häufig } \\
(\%)\end{array}$ & $\begin{array}{l}\text { Manchmal } \\
(\%)\end{array}$ & $\begin{array}{l}\mathrm{Nie} \\
(\%)\end{array}$ & $N$ & $\begin{array}{l}\text { Häufig } \\
(\%)\end{array}$ & $\begin{array}{l}\text { Manchmal } \\
(\%)\end{array}$ & $\begin{array}{l}\mathrm{Nie} \\
(\%)\end{array}$ \\
\hline$P M \_M A$ & 138 & 74,60 & 21,00 & 4,30 & 132 & 67,40 & 22,70 & 9,80 \\
\hline$P M \_P I$ & 127 & 60,60 & 20,50 & 18,90 & 103 & 57,30 & 37,90 & 4,90 \\
\hline$B A \_P O$ & 392 & 98,20 & 1,50 & 0,30 & 391 & 95,10 & 3,80 & 1,00 \\
\hline$B A \_B V$ & 310 & 99,70 & 0,30 & 0,00 & 310 & 94,50 & 4,80 & 0,60 \\
\hline $\begin{array}{l}D E \\
\text { alle }\end{array}$ & 20.009 & 77,14 & 11,88 & 10,98 & 17.813 & 75,19 & 16,10 & 8,72 \\
\hline
\end{tabular}

Tab. 3 KI- und Kontext-Kompetenzen

\begin{tabular}{|c|c|c|c|c|c|c|c|c|c|}
\hline \multicolumn{5}{|c|}{ KI/ML-Kompetenz } & \multicolumn{5}{|c|}{ Kontext-Kompetenz (AV-Index) } \\
\hline & $N$ & $\begin{array}{l}\text { Light } \\
(\%)\end{array}$ & $\begin{array}{l}\text { Hoch } \\
(\%)\end{array}$ & $\begin{array}{l}\text { Keine } \\
(\%)\end{array}$ & $N$ & MW & SD & $\mathrm{AV}>0,5 \mathrm{~N}$ & $\begin{array}{l}\mathrm{AV}>0,5 \\
(\%)\end{array}$ \\
\hline$P M \_M A$ & 106 & 10,60 & 3,30 & 86,20 & 126 & 0,71 & 0,222 & 114 & 90,48 \\
\hline$P M \_P I$ & 94 & 10,70 & 5,40 & 83,90 & 111 & 0,65 & 0,206 & 91 & 81,98 \\
\hline$B A \_P O$ & 299 & 17,40 & 2,70 & 79,90 & 359 & 0,67 & 0,180 & 310 & 86,35 \\
\hline$B A \_B V$ & 220 & 19,20 & 2,50 & 78,30 & 272 & 0,70 & 0,165 & 247 & 90,81 \\
\hline $\begin{array}{l}D E \\
\text { alle }\end{array}$ & 18.768 & 10,65 & 3,97 & 85,37 & 17.339 & 0,58 & 0,271 & 13.001 & 74,98 \\
\hline
\end{tabular}

beschäftigt. Beide Beschäftigtengruppen im Kontext PM sind damit deutlich männlicher, leicht jünger und haben etwas mehr Erfahrungsjahre als der Durchschnitt der Beschäftigten in Deutschland (vgl. Tab. 1, 2 und 3).

\subsection{Kontext beratungsintensive Sachbearbeitung}

Auch hier wird zunächst ein erster Kategorisierungsschritt über Berufe: vollzogen: Ausgangspunkt sind die zwei unternehmensintern wirkenden 3-Steller der KldB 2010 für Personalwesen und -dienstleistung (715) mit $N=231$ und für Unternehmensorganisation und -strategie (713) mit $N=996$ einerseits, sowie die eher nach Außen wirkenden Berufe der Versicherungs- und Finanzdienstleistungen (721) mit $N=537$ andererseits. Für den zweiten Kategorisierungsschritt über Tätigkeiten wird hier dann kontextrelevantes Wissen angenommen, wenn zusätzlich die Tätigkeit „Beraten und informieren“(F314) als häufig angegeben wird. Auch in diesem Kontext wird als dritter Kategorisierungsschritt das Anforderungsniveau und erneut bewusst nur die beruflich erst- und weitergebildeten Beschäftigten herangezogen. Dies hat zwei Gründe: Einerseits ist die übliche Zuschreibung, dass deren Tätigkeit im Vergleich zu akademischem Anforderungsprofil standardisierter und daher leichter durch KI/ML-basierte Automatisierung zu ersetzen sei. Andererseits kann davon ausgegangen werden, dass Beschäftigte auf diesem Anforderungsniveau aktuell noch mehr im direkten Kontakt mit ihren Kund*innen stehen und daher näher am Kontext sind. 
Entsprechend diesen Kategorisierungsschritten umfassen die Kontextbeschäftigten in den beiden Bereichen der beratungsintensiven Sachbearbeitung (BA) ,Personal/Orga “ $N=392$ (nachfolgend BA_PO) und ,Bank/Versicherung “ $N=310$ (nachfolgend $B A \_B V$ ). Wenig überraschend finden sich hier mehr Frauen: (64\% bei PO und $51,6 \%$ bei BV). Die Beschäftigten sind im Durchschnitt 46,6 (PO; SD = 10,85) und 47,1 (BV; SD=9,76) Jahre alt und durchschnittlich mit 9,9 (PO; SD=8,27) bzw. 13,1 (BV; SD=9,45) etwas kürzer in ihrer aktuellen Tätigkeit beschäftigt als die Beschäftigten im Kontext Instandhaltung. Die Beschäftigtengruppen im Kontext BA sind damit weiblicher, ähnlich alt und haben teils etwas mehr Erfahrungsjahre als der Durchschnitt der Beschäftigten in Deutschland (vgl. Tab. 1, 2 und 3). Nach diesen ersten kategorisierenden Auswertungen ist der Anteil der im Fokus stehenden Beschäftigten in den beiden Bereichen Predictive Maintenance und beratungsintensive Sachbearbeitung sichtbar gemacht und kann nun nach den vorhandenen, KI/MLrelevanten Kompetenzen analysiert werden.

\section{KI und Kontext: Kompetenzen der Beschäftigten - Potenzial oder Hürde?}

Zunächst liegt nahe, die Kompetenzen zum Umgang mit KI/ML in Bezug auf den Digitalisierungsgrad des Arbeitsplatzes zu betrachten. Wie viele Massendatensätze hinkt die BIBB/BAuA-Erwerbstätigenbefragung aber gerade in der Erfassung der digitalen Arbeitsumgebung dem Stand und der Differenziertheit der Software-Anwendungen, Tools und Gadgets am Arbeitsplatz völlig hinterher. Für die beiden Kontextgruppen lässt sich im ersten Zugriff Digitalisierung daher nur sehr grob betrachten: Während alle Beschäftigten in Deutschland zu 77,1\% angeben, häufig am Computer zu arbeiten, arbeiten die Beschäftigten im Kontext BA mit bis zu 99,7\% ganz überwiegend häufig am Computer. Die Beschäftigten im Kontext PM sind dagegen mit 60,6-74,6\% seltener häufig am Computer. Insbesondere Beschäftigte im Bereich PM-PI geben noch zu 18,9\% an, nie am Computer zu arbeiten. Dass diese Gruppe aber deswegen nicht deutlich weniger digital unterwegs ist, zeigen die Werte zur Nutzung von Internet und E-Mail (vgl. Tab. 1, 2 und 3) - nur 4,9\% der gleichen Gruppe geben an, dies nie zu tun - offensichtlich ist hierbei aber nicht der Rechner entscheidend, sondern wohl verschiedene mobile Endgeräte (nach denen die Erhebung aber leider nicht fragt).

Will man eine Einschätzung zu vorhandenen KI-Kompetenzen der Beschäftigten kriegen, hilft also diese Annäherung über die Digitalisierung nicht weiter. Die BIBB/BAuA-Erwerbstätigenbefragung ermöglicht jedoch über Variablen zu konkreten Anforderungen am Arbeitsplatz, mögliche vorhandenene Kompetenzen sichtbar zu machen. Die empirische Analyse konzentriert sich bewusst nicht darauf, ob einschlägige Kenntnisse irgendwann über formale Bildungsprozesse erworben wurden, sondern einschlägige Kenntnisse und Fähigkeiten, die lebendig sind, weil sie am aktuellen Arbeitsplatz aktiv abgefordert werden. Wo dies der Fall ist - so die These - sollte für eine Weiterentwicklung und selbst ein Training on the Job in Richtung KI-Anwendung und -Verständnis relativ leicht anzuknüpfen sein (Abb. 1). 

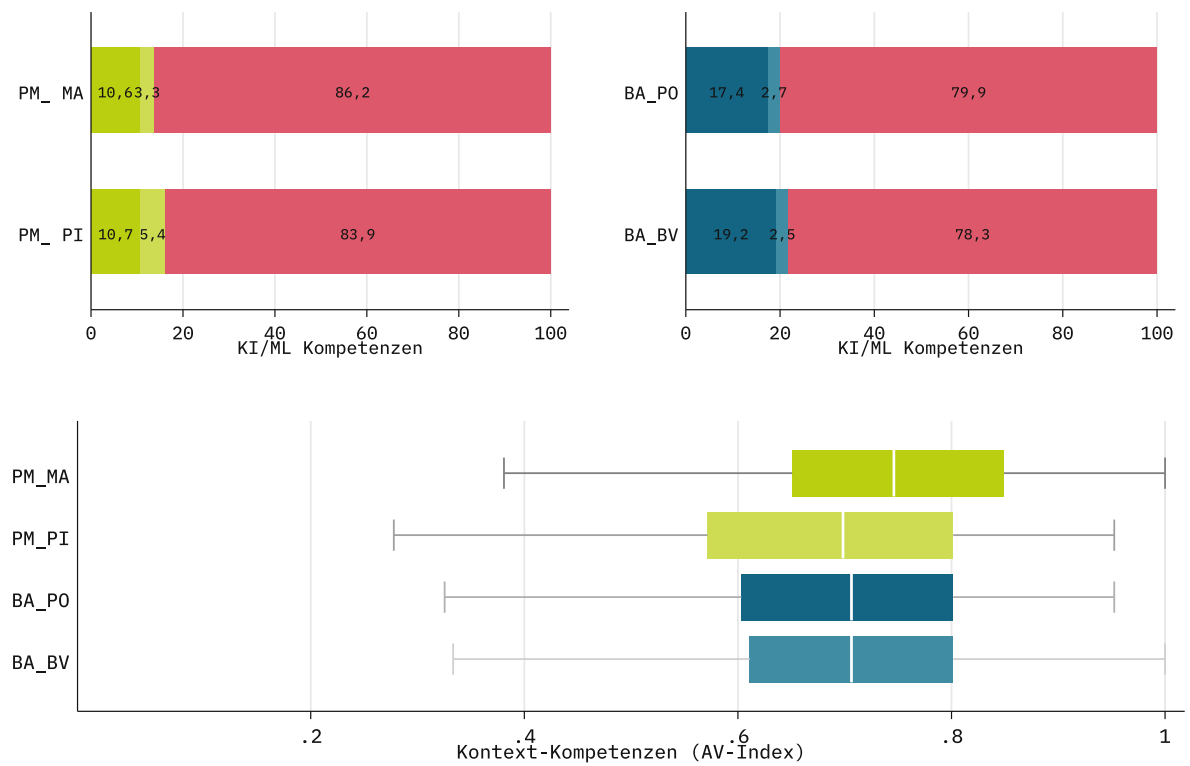

Abb. 1 Potenziale der Beschäftigten für KI/ML und Kontext

Für beide Bereiche sind diese Angaben zur Häufigkeit der Computernutzung aber nicht hinreichend, um der Frage nachzugehen, die hier verfolgt wird, nämlich: Welche Potenziale sind bei den Beschäftigten vorhanden, um sinnvolle KI/ML-Anwendungen zu implementieren und auf Dauer produktiv nutzbar zu machen? Es geht also um die Frage: kann KI/ML erfolgreich umgesetzt werden oder sind die dafür nötigen KI- und Kontext-Kompetenzen noch nicht bei den Beschäftigten vorhanden. Um dieser Frage nachzugehen werden nachfolgend zwei Kompetenz-Dimensionen betrachtet:

- Erstens sind Beschäftigte hilfreich, die elaboriertere IT-Kenntnisse verbinden können mit einem tiefergehenden mathematischen und/oder statistischen Verständnis. Diese könnten ohne großen Aufwand in Richtung Data Science weiterentwickelt werden und brächten nutzbares Wissen bereits mit, das etwa zur Auswahl passender Algorithmen benötigt wird. Um dieses Potenzial der Beschäftigten für das Verständnis und den Umgang mit KI/ML-basierten Technologien zu erfassen, werden mehrere Anforderungs- und Tätigkeitsvariablen der BIBB/ BAuA-Erwerbstätigenbefragung kombiniert (3.1.).

- Zweitens wird die Bedeutung des Anwendungskontexts betrachtet und diesem in doppelter Hinsicht Rechnung getragen: Wo Beschäftigte heute noch mit Wandel, Komplexität und Unwägbarkeiten häufig umgehen müssen, stoßen heutige KI/ML-Anwendungen schnell an ihre Grenzen und brauchen zwingend für einen sinnvollen Einsatz die Kontextualisierung durch den Menschen (während der KI/ML-Einführung sowie ihrer dauerhaften Anwendung). Beschäftigte in diesen Kontexten bringen - gerade, weil sie mit Wandel, Komplexität und Unwägbarkeiten umgehen - dieses unverzichtbare Potenzial mit. Wie weit es in den hier 
betrachteten Anwendungsgebieten von KI/ML bei den Beschäftigten als Kompetenz bereits vorhanden ist, zeigt der zweite Analyseschritt (3.2.).

\subsection{KI-Kompetenz: Statistik- und Programmiererfahrung}

Um die hier verfolgtekompetenzorientierte Analyse vorzunehmen, werden zunächst Kompetenzen für KI/ML betrachtet; dabei geht es um die Frage wie viele Beschäftigte an ihrem Arbeitsplatz bereits heute Fachwissen und Fähigkeiten benötigen, die das Verständnis und eine Weiterbildung in Richtung KI/ML-erleichtern sollten. Eine kategoriale Annährung an diese wird über vier Tätigkeitsitems vorgenommen, die den Umgang mit Daten sowie statistische und IT-Fachkenntnisse bündeln. Als KI/ML-Kompetenz wird dabei auf Basis der Variablen der BIBB/BAuA-Erwerbstätigenbefragung definiert, wenn Beschäftigte

- erstens häufig „Informationen sammeln, recherchieren, dokumentieren“ (F313),

- und zweitens angeben, im Bereich „Mathematik, Fachrechnen, Statistik (F403_04)“" nicht nur Grund-, sondern Fachkenntnisse zu benötigen;

- zudem zum Dritten am Arbeitsplatz Fachkenntnisse in PC-Anwendungsprogrammen (F403_06) benötigen;

- sowie viertens nicht nur IT anwenden, sondern einen elaborierteren IT-Umgang (F324) braucht (etwa Programmieren, Systemadministration o. Ä.).

Sind diese vier Anforderungen am Arbeitsplatz aktuell schon gegeben, ist ein Bündel an lebendigen Fähigkeiten und Kenntnissen vorhanden, die einen Einstieg in die Thematik KI/ML deutlich erleichtern sollten - hier kann also von einem KI/MLFachkompetenz ausgegangen werden. Beschäftigte, die bei den Punkten 1 bis 3 dieselben Angaben machen, aber IT lediglich anwenden, bringen zwar einschlägiges Potenzial im Hinblick auf den Umgang mit Daten und statistischen Verfahren mit, benötigen aber mehr Einführung bzw. Weiterbildung im Bereich elaborierterer ITAnwendung. Wir bezeichnen dieses Potenzial nachfolgend als KI/ML-Basiskompetenz. Sehr vorsichtig wird schließlich für alle Beschäftigten dann keine vorhandene KI/ML-Kompetenz angenommen, die bei mindestens einer der Tätigkeiten 1 bis 3 angeben, am Arbeitsplatz lediglich Grund- oder keine Kenntnisse zu benötigen (was nicht bedeutet, dass ihnen nicht ebenfalls Grund- oder Fachkenntnisse im Umgang mit KI/ML nahe gebracht werden könnten). Damit soll nicht behauptet werden, dass die Beschäftigten mit KI/ML-Fach- oder Basiskompetenzen bereits KI/ML-spezifische Kenntnisse ausgeprägt haben. Es geht hier um die quantitative Einschätzung für bestehende Kompetenzen, die eine Aneignung KI/ML-spezifischen Wissens und Verständnisses deutlich erleichtern sollten. Aus Unternehmens- und Managementsicht lohnt es sich, Beschäftigte mit diesem Potenzial im eigenen Unternehmen zu identifizieren.

Die Analysen zeigen, dass nach dieser Kategorisierung in Deutschland fast $4 \%$ aller Beschäftigten bereits KI/ML-Fachkompetenzen und weitere 10,7 \% ein KI/MLBasiskomptenezen (vgl. Tab. 1, 2 und 3); rd. 14,6\% der Beschäftigten bringen also aktiv im Arbeitsalltag benötigte Kenntnisse und Fähigkeiten mit, die ein solides Fundament für das grundlegende Verständnis für KI/ML und/oder für eine entspre- 
chende Vertiefung bilden. Kein so schlechter Ausgangswert für eine Technologie, die an den meisten Arbeitsplätzen spürbar noch gar nicht angekommen ist.

In den betrachteten Kontexten, in denen aktuell die praktische Anwendung von $\mathrm{KI} / \mathrm{ML}$ im Unternehmen am deutlichsten voran getrieben wird, zeigt sich ein durchwachsenes Bild: Teils ist die Ausgangslage besser, teils schlechter als in der gesamten Erwerbstätigenstruktur. Bei Banken und Versicherungen (BA_BV) etwa findet sich der höchste Wert an KI/ML-Basis-Potenzial: 19,2\% der Beschäftigten benötigen auch heute schon die dafür nötigen Fachkompetenzen im Bereich Mathematik/ Statistik und der Bedienung von entsprechenden IT-Anwendungsprogrammen. Interessanterweise sind hier aber nur 2,1\% Beschäftigte in der Lage, zusätzlich mit IT auch jenseits der reinen Anwendung umzugehen, das ist nicht nur ein geringerer Anteil als bei den anderen betrachteten Kontexten, sondern er ist auch geringer als bei allen Beschäftigten in Deutschland. Eine ähnliche Schere findet sich im Bereich HR und Unternehmensorganisation: Auch hier ein mit 17,4\% hoher Anteil an KI/ML-Basiskompetenzen gepaart mit einem unterdurchschnittlichen Anteil von $2,7 \% \mathrm{KI} / \mathrm{ML}-$ Fachkompetenzen. Es fehlt also in beiden Bereichen der beratungsintensiven Sachbearbeitung an tiefergehenden IT-Kenntnissen.

Bei den beiden Kontexten für Predictive Maintenance stellt sich das Bild andersherum dar. Der im Vergleich höchste Anteil an KI/ML-Fachkompetenzen findet sich dagegen mit 5,4\% am deutlichsten ausgeprägt im Kontext Prozesse/Infrastruktur (PM_PI; bei einem Basiskompetenz-Anteil von 10,7\%). Dieser liegt mit 10,6\% im Bereich PM_MA fast gleich auf, hier ist der Anteil von Beschäftigten mit einschlägigen Fachkompetenzen mit 3,3\% zwar leicht geringer als der Beschäftigtendurchschnitt, aber auch leicht höher als in den Sachbearbeitungsgruppen.

\subsection{Kontext-Kompetenz: Wandel, Komplexität und Unwägbarkeiten}

Beim aktuellen Stand von KI/ML-Technologien ist davon auszugehen, dass die aus diesen Systemen generierten Prognosen in einem stark von Wandel, Komplexität und Unwägbarkeiten geprägten Kontext schnell an ihre Grenzen kommen und in besonderen Maße eine Re-Kontextualisierung durch die Beschäftigten benötigen zumindest dann, wenn die Prognosen und Entscheidungsempfehlungen der Systeme sich als produktiv und robust und nicht als sinnentleerte Artefakte ohne praktischen Nutzen und ggf. sogar mit Schadenspotenzial erweisen sollen. Beschäftigte, die heute schon mit Wandel, Komplexität und Unwägbarkeiten täglich umgehen, wissen um jene Bereiche ihres Arbeitsumfelds, mit der sich KI/ML-Algorithmen generell schwertun: Nämlich die Unterscheidung von Fehlmessung und echtem d.h. sachlich relevantem - Ausreißer. Beschäftigte, die sich in einem komplexen und vielfältigen Kontext bewegen, bringen besondere Kompetenzen für die Kontextualiserung von Algorithmen und Lerndaten mit, denn ihre Kompetenz ist eben nicht nur die Prognose aus vergangenen Erfahrungen, sondern auch die Fähigkeit zum Handeln angesichts bislang nicht aufgetretener Fehler.

Dieses Fähigkeitsbündel lässt sich mit Daten der BIBB/BAuA-Erwerbstätigenbefragung gut abbilden, um die aktuelle Kontextualisierungsanforderung am Arbeitsplatz zu erfassen wird der AV-Index (AV = Arbeitsvermögen $)$ verwendet. Basis dieser bereits etablierten und vielfältig angewendeten Indexbildung ist ein in Jahrzehnten 
kumulierter Forschungsstand der qualitativen Arbeitsforschung, zudem wurde der Index mehrfach und u. a. ökologisch validiert (vgl. Pfeiffer 2018a). Der aus $17^{2}$ Tätigkeitsitems generierte AVI bildet ab, wie häufig an einem Arbeitsplatz mit Wandel, Komplexität und Unwägbarkeiten umgegangen werden muss und ist damit ein guter Indikator für die Grenzen des Einsatzes von KI/ML an einem Arbeitsplatz wie für das Potenzial der Beschäftigten, KI/ML-Technologien für einen sinnvollen Einsatz mitzugestalten. Ein hoher AV-Wert verweist also ebenso auf starke Anforderungen zur Rekontextualisierung einzusetzender Algorithmen wie auf die bestehenden Kompetenzen der Beschäftigten in diesen Bereichen für eben diesen Kontextbezug. Der Mittelwert des AV-Index liegt für alle Beschäftigten bei 0,58 ( $\mathrm{SD}=0,271$; $N=17.339$ ). Insgesamt weisen $75 \%$ aller Beschäftigten in Deutschland überdurchschnittlich hohe AV-Index-Werte auf, d.h. die Mehrheit ist an ihrem Arbeitsplatz häufig mit Wandel, Komplexität und Unwägbarkeiten konfrontiert - oder anders formuliert: bewältigt und „kann“ Kontext. Der AV-Index wird hier als Kompetenz der Beschäftigten für die Kontextualisierung von KI/ML in konkreten Anwendungsbereichen interpretiert - und zeigt gleichzeitig an, wie kontextualisierungsbedürftig diese Bereiche sind.

Die Werte der hier untersuchten Kontexte zeigen im Vergleich zu allen Beschäftigten in Deutschland höhere Anforderungen im Umgang mit Wandel, Komplexität und Unwägbarkeiten. Einen Spitzenwert von 0,71 haben dabei die Beschäftigten im Bereich Predictive Maintenance/MuE - Automotive (PM_MA). Auch die anderen drei Kontextgruppen erreichen überdurchschnittlich hohe AV-Indexwerte: BA_BV liegen mit 0,70 knapp dahinter, gefolgt von PM_PI und BA_PO mit 0,65 und 0,67 (vgl. Tab. 1, 2 und 3 sowie zu Lage und Streuung die Boxplots in der Abb. 1).

\section{KI und Kontext: Das Potenzial ist da}

Wo immer es um die Erfassung und Auswertung von Unternehmensdaten ging gilt schon seit langem die Aufforderung: „If it matters, measure it!“ (SingletonGreen 1993). Ganz ähnlich sehen das wohl auch alle, die mit Big Data und KI/ML arbeiten. Das grundsätzliche Problem ist, dass wir nicht immer wissen können, was wichtig ist - geschweige denn, was unter welchen Umständen für wen in Zukunft wichtig sein könnte. Leicht verschwindet hinter den Daten die Tatsache, dass jede datenförmige Erfassung immer nur eine Abstraktion von Welt ist, ein Ausschnitt einer viel komplexeren Wirklichkeit - und noch gravierender: wir wissen nicht in welchem Verhältnis der von uns zu einem bestimmten Zeitpunkt realisierbare Datenausschnitt zur realen Welt dahinter steht. Verliert sich dies aus dem Blick,

\footnotetext{
2 Zur Konstruktion des AVI vgl. (Pfeiffer 2018b). Der Index wird aus drei Teilindices (situative Unwägbarkeiten, strukturelle und situative Komplexität) gebildet, die Notwendigkeit einer längeren Einarbeitungszeit gilt als Multiplikator. Eine Faktorenanalyse (Pfeiffer 2018a) zur Überprüfung der Konstruktvalidität der 18 Items des AVI zeigte ein Item mit besonders geringer Faktorladung und sehr geringer Kommunalität. Dieses Item zur Häufigkeit mit der,,auch schon ein kleiner Fehler oder eine geringe Unaufmerksamkeit größere finanzielle Verluste zur Folge haben“ kann (F411_11) wird daher in den neueren Berechnungen des AVI nicht mehr herangezogen.
} 
dreht sich das Motto Singleton-Greens schnell um in ein: „We measure it, so it must matter".

Schon Mitte der 1980er Jahre diskutiert die wissenschaftliche KI-Community Themen, die sich leicht in die heutige Zeit verlängern lassen: Erstens überzogene Erwartungen an die KI; zweitens die Vorstellung des Managements, dass KI schnell in produktive Systeme zu übersetzen sei; drittens dass es deutlich mehr wissenschaftlicher und erkenntnistheoretischer Forschung zu KI bedürfe statt immer neuer Anwendungen und schließlich viertens, dass dafür kaum Personal für die Universitäten zu finden sei, weil Unternehmen, Start-ups und Venture Kapitalisten deutlich attraktivere Perspektiven böten (vgl. McDermott et al. 1985).

Manches hat sich seither verändert (so reden wir nicht mehr von Expertensystemen oder der Programmiersprache LISP), anderes klingt erstaunlich aktuell. Auch heute findet sich die Vorstellung, KI/ML seien vergleichsweise einfach anzuwendende, gleichzeitig beeindruckend mächtige Tools. Anders ist heute auch die Rechenleistung und die Existenz großer Datenbestände - von Big Data, über Data Warehouses bis Data Lakes. Das weckt Erwartungen an neue Geschäftsmodelle. Oft erwartet das Management, dass aus der Kombination von vielen Daten mit (irgend-)einem KI/ML-Algorithmus quasi automatisch ein bahnbrechender Outcome generiert wird. Dies lässt sich gerade in vielen Unternehmen beobachten. Unterschätzt wird dabei zum einen die Qualität der Daten: Diese liegen in unterschiedlichen Formaten und mehr oder weniger strukturiert vor, sie können von dynamischer oder statischer Natur sein, sie können Prozessdaten (z. B. Nebenprodukte bestimmter Verwaltungsvorgänge) sein oder empirische Daten - bei diesen Fragen aber fängt die Problematik erst an. In den meisten Unternehmen wird die nächste Ebene der notwendigen Fragen - etwa die nach Skalenniveau oder Verteilungsformen - schon erschreckend häufig nicht mehr gestellt.

Zwar gibt es in der KI-Community lebhafte Debatten, ob die alte Frage nach dem Zusammenhang von statistischer Korrelation und realer Kausalität bei algorithmischen Ansätzen (im Gegensatz zu modellbasierter Statistik) nicht obsolet sei (Breiman 2001; Anderson 2008). Vergessen wird dabei, dass der eine wie andere Angang eigentlich nur eines leisten kann: Hinweise geben auf mögliche Zusammenhänge, die über andere Formen der Beobachtung nicht zu bemerken sind. Vergessen wird dabei auch, dass durch ein Anwachsen von (u. U. irrelevanten) Variablen, das damit einher gehende Datenrauschen vermehrt zu falschen Prognosen führen kann (Ng und Soo 2018, S. 5). Ob es diese Zusammenhänge gibt (und welche Wirkmechanismen auf der Mikroebene dahinter liegen), dies sind Fragen, die der noch so große Datensatz, der noch so treffsichere Algorithmus und die noch so gute Vorhersage nicht beantworten. Antworten darauf können nur außerhalb der Daten gesucht und wenn es sie denn gibt - gefunden werden. Hier aber könnten echte Antworten oder vielleicht auch nachhaltig tragende Geschäftsmodelle verborgen liegen. Umgekehrt gilt es, sich bewusst zu machen, dass die Gefahr eines ökologischen Fehlschlusses in der Welt von KI/ML nicht ausgeschlossen, sondern potenziert ist. Das mag vernachlässigbar sein, wenn es um Prognosen zum individuellen Einkaufsverhalten geht; das kann aber zu dramatischen Risiken führen, wenn wir von laufender Produktion, stabilen Logistik- und Infrastrukturprozessen oder lebensrettenden medizinischen Diagnosen reden (mit teils auch ökonomisch desaströsen Folgen). Wer sich profes- 
sionell und nicht nur oberflächlich mit KI/ML beschäftigt, weiß um diese Probleme. Im ,hands on“-Einsatz von KI/ML-Tools wie er im Moment in vielen Unternehmen zu beobachten ist, sind den Beteiligten die vielfältigen Grenzen ihres Tuns oft nicht bewusst.

Ziel dieses Artikels war, die Potenziale für den Einsatz von KI/ML im Unternehmen mit Blick auf die Kompetenzen der Beschäftigten empirisch zu prüfen. Die auch von Unternehmen vordergründig wahrgenommene Grenze ist der viel beklagte Mangel an Expert*innen für die Programmierung der Algorithmen oder DataScientists für die Aufbereitung von Lerndatensets. Das aber ist nur die eine Anforderung an das nötige Humankapital. Die hier vorgestellten Daten zeigen welche Kompetenzen bei den sozusagen ganz normalen Beschäftigten vorhanden sind, die dabei helfen können, KI/ML in einem produktiven und robusten Einsatz zu bringen. Für die untersuchten Bereiche Predictive Maintenance und beratungsintensive Sachbearbeitung wurden dabei zwei Kompetenzfelder unterschieden: Für KI/ML-Expertenwissen finden sich als Ausgangsbasis erstens hilfreiche Kompetenzen bei den Beschäftigten, die heute schon aktiv am Arbeitsplatz mit Statistikfachkenntnissen und elaborierten IT-Anwendungen umgehen, sie wissen um die immanenten Grenzen datenbasierter Mustererkennung und von Wahrscheinlichkeitsaussagen. Sie lassen sich damit leichter zu KI/ML-Expert*innen weiter entwickeln, als Beschäftigte ohne derart aktives Wissen. Von diesen Beschäftigten, das konnte hier empirisch gezeigt werden finden sich relevante Anteile innerhalb der Anwendungskontexte, in denen KI/ML gerade in den Unternehmen praktisch wird. Beschäftigte, die Grundlagen (wie lebendige Fachkenntnisse in Mathematik und Statistik und über die reine Anwendung hinausgehende IT-Nutzung) für ein gutes KI/ML-Verständnis bereits mitbringen - und zudem den Kontext der Anwendung kennen wären mit wenig Aufwand in Richtung KI/ML weiterzubilden und dabei schneller in der Lage, lauffähige und sinnvolle KI/ML-Anwendungen zu entwickeln als dies bspw. Absolvent*innen aus IT-Studiengängen ohne Statistikwissen und ohne praktische Kontexterfahrungen gelingen kann.

Die Ergebnisse der hier vorgestellten Auswertungen zeigen zweitens, dass die Kontexte Predictive Maintenance und beratungsintensive Sachbearbeitung komplexer, wandlungsfähiger und unplanbarer sind als üblicherweise angenommen. Das ist an sich kein neuer Befund, er wird dennoch in der Unternehmenspraxis oft vernachlässigt: Management wie IT-Entwicklung neigen oft genug zu einer Unterschätzung dieser Komplexität. Die vorgestellten Analysen mit Hilfe des AV-Index belegen, dass die Beschäftigten in den untersuchten Kontexten im hohen Maße mit diesen Unwägbarkeiten umgehen. Sollen KI/ML-Anwendungen dort sinnvoll, robust und produktiv zum Einsatz kommen, müssen die immanenten Grenzen von KI/ML kompensiert werden - durch Menschen, die diesen Kontext nicht nur kennen, sondern tagtäglich bewältigen. Dieses Potenzial der Beschäftigten findet sich in den untersuchten Bereichen überdurchschnittlich. Das Potenzial für KI und Kontext ist also durchaus vorhanden. Die Herausforderung an das Management ist daher vor allem: a) die Grenzen und Herausforderungen zu verstehen und ernstzunehmen, die mit KI/ML einhergehen; b) die Beschäftigten mit KI/ML-Kompetenzen zu identifizieren und entsprechend weiterzubilden und c) die Beschäftigten mit besonders hohem Kontext-Wissen systematisch und frühzeitig in die Teams und Prozesse zur Umset- 
zung von KI/ML-Anwendungen zu integrieren. Eigentlich Binsenweisheiten, die in der Unternehmenspraxis jedoch allzu oft nicht befolgt werden.

Funding Open Access funding provided by Projekt DEAL.

Open Access Dieser Artikel wird unter der Creative Commons Namensnennung 4.0 International Lizenz veröffentlicht, welche die Nutzung, Vervielfältigung, Bearbeitung, Verbreitung und Wiedergabe in jeglichem Medium und Format erlaubt, sofern Sie den/die ursprünglichen Autor(en) und die Quelle ordnungsgemäß nennen, einen Link zur Creative Commons Lizenz beifügen und angeben, ob Änderungen vorgenommen wurden.

Die in diesem Artikel enthaltenen Bilder und sonstiges Drittmaterial unterliegen ebenfalls der genannten Creative Commons Lizenz, sofern sich aus der Abbildungslegende nichts anderes ergibt. Sofern das betreffende Material nicht unter der genannten Creative Commons Lizenz steht und die betreffende Handlung nicht nach gesetzlichen Vorschriften erlaubt ist, ist für die oben aufgeführten Weiterverwendungen des Materials die Einwilligung des jeweiligen Rechteinhabers einzuholen.

Weitere Details zur Lizenz entnehmen Sie bitte der Lizenzinformation auf http://creativecommons.org/ licenses/by/4.0/deed.de.

\section{Literatur}

Anderson C (2008) The end of theory: the data deluge makes the scientific method obsolete. In: WIRED. https://www.wired.com/2008/06/pb-theory/. Zugegriffen: 4. Aug. 2019

Breiman L (2001) Statistical modeling: The two cultures. Statistical Science 16:199-231. https://doi.org/ $10.1214 / \mathrm{ss} / 1009213726$

Debortoli S, Müller O, vom BJ (2014) Vergleich von Kompetenzanforderungen an Business-Intelligenceund Big-Data-Spezialisten. Wirtschaftsinf 56:315-328. https://doi.org/10.1007/s11576-014-0432-4

Dengler K, Matthes B (2015) Folgen der Digitalisierung für die Arbeitswelt.Substituierbarkeitspotenziale von Berufen in Deutschland. IAB, Nürnberg

Doleski OD (2020) Energieversorgungsunternehmen neu denken: Utility 4.0. In: Doleski OD (Hrsg) Realisierung Utility 4.0 Band 1: Praxis der digitalen Energiewirtschaft von den Grundlagen bis zur Verteilung im Smart Grid. Springer, Wiesbaden, S 3-62

Elert F (2019) Digitalisierung des Geschäftsmodells Versicherung - Potenziale von digitalen AssistanceDienstleistungen. In: Reich M, Zerres C (Hrsg) Handbuch Versicherungsmarketing. Springer, Berlin, Heidelberg, S 219-241

Frey CB, Osborne MA (2017) The future of employment: how susceptible are jobs to computerisation? Technol Forecast Soc Change 114:254-280. https://doi.org/10.1016/j.techfore.2016.08.019

Garcia E, Costa A, Palanca J et al (2020) Requirements for an intelligent maintenance system for industry 4.0. In: Borangiu T, Trentesaux D, Leitão P et al (Hrsg) Service oriented, Holonic and multi-agent manufacturing systems for industry of the future. Springer, Cham, S 340-351

Helmrich R, Tiemann M, Troltsch K et al (2016) Digitalisierung der Arbeitslandschaften. Keine Polarisierung der Arbeitswelt, aber beschleunigter Strukturwandel und Arbeitsplatzwechsel. Bundesinstitut für Berufsbildung (BIBB), Bonn

Lemmer S, Schlereth T, Eser A, Klußmann S (2019) Wie die Digitalisierung HR und AI von Antipoden zu Partnern macht. Digit Welt 3:46-55. https://doi.org/10.1007/s42354-019-0194-7

Levy F, Murnane RJ (2005) The new division of labor: how computers are creating the next job market. SAGE, Princeton, Oxfordshire

McDermott D, Waldrop MM, Schank R et al (1985) The dark ages of AI: a panel discussion at AAAI-84. Ai Mag 6:122-134

Ng A, Soo K (2018) Data Science - was ist das eigentlich?! Algorithmen des maschinellen Lernens verständlich erklärt. Springer, Berlin

Pfeiffer S (2018a) Die Quantifizierung von Nicht-Routine. Zur ökologischen Validierung des Arbeitsvermögen-Index - und einem anderen Blick auf das Ersetzungspotenzial von Produktionsarbeit. Arb Zeitschrift Für Arbeitsforsch Arbeitsgestaltung Arbeitspolitik 27:213-237. https://doi.org/10.1515/ arbeit-2018-0018 
Pfeiffer S (2018b) The 'future of employment' on the shop floor: why production jobs are less susceptible to computerization than assumed. Int J Res Vocat Educ Train 5:208-215. https://doi.org/10.13152/ IJRVET.5.3.4

Reich M, Blodau T (2019) Frühwarnung im Kundenbindungsmanagement von Versicherungen. In: Reich M, Zerres C (Hrsg) Handbuch Versicherungsmarketing. Springer, Berlin, Heidelberg, S 627-645

Rohrbach-Schmidt D, Hall A (2020) BIBB/BAuA-Erwerbstätigenbefragung 2018. Version 1.0. Bundesinstitut für Berufsbildung, Bonn

Singleton-Green B (1993) If it matters, measure it! In: Accountancy, S 52-53

Tallgauer M, Festing M, Fleischmann F (2020) Big Data im Recruiting. In: Verhoeven T (Hrsg) Digitalisierung im Recruiting: Wie sich Recruiting durch künstliche Intelligenz, Algorithmen und Bots verändert. Springer, Wiesbaden, S 25-39

Timofeev AV, Denisov VM (2020) Machine learning based predictive maintenance of infrastructure facilities in the cryolithozone. In: Pricop E, Fattahi J, Dutta N, Ibrahim M (Hrsg) Recent developments on industrial control systems resilience. Springer, Cham, S 49-74

Zacherl V, Peters A, Weber S (2019) Einsatzfelder Künstlicher Intelligenz in der Finanzdienstleistung. Universität Regensburg, Regensburg

Zhao Y, Hryniewicki MK, Cheng F et al (2019) Employee turnover prediction with machine learning: a reliable approach. In: Arai K, Kapoor S, Bhatia R (Hrsg) Intelligent systems and applications. Springer, Cham, S 737-758

Zschech P, Fleißner V, Baumgärtel N, Hilbert A (2018) Data science skills and enabling enterprise systems. HMD 55:163-181. https://doi.org/10.1365/s40702-017-0376-4 